\title{
IDENTIFICAÇÃO DE FONTES DE RESISTÊNCIA EM FEIJOEIRO A Sclerotium rolfsii
}

\section{SUZANA A.F. DANTAS, SÔNIA M.A. OLIVEIRA, RILDO S.B. COELHO \& ROBERTO L. X. SILVA}

Universidade Federal Rural de Pernambuco, Departamento de Agronomia-Fitossanidade, 52171-900, Recife-PE, e-mail: suzanaalencar@globo.com

(Aceito para publicação em 29/07/2002)

Autor para correspondência: Suzana A.F. Dantas

DANTAS, S.A.F., OLIVEIRA, S.M.A., COELHO, R.S.B. \& SILVA, R.L.X. Identificação de fontes de resistência em feijoeiro a Sclerotium rolfsii. Fitopatologia Brasileira 27:528-531. 2002.

\section{RESUMO}

A podridão do colo do feijoeiro comum (Phaseolus vulgaris), causada por Sclerotium rolfsii, pode ocasionar grandes perdas na produção da cultura, já que as medidas de controle aplicadas para esta doença, não têm proporcionado resultados efetivos. Neste contexto, existe a necessidade de identificar fontes de resistência em cultivares e linhagens de feijoeiro a $S$. rolfsii. Os isolados foram obtidos de caules de plantas de feijoeiro infetadas, provenientes de diferentes sub-regiões do Estado de Pernambuco e a inoculação foi realizada por deposição de dez escleródios no colo das plantas, previamente ferido. A severidade da doença foi avaliada através de escala de notas variando de 1 a 9, proposta pelo CIAT, e os dados transformados para índice de doença de Mackinney. Todas as cultivares e linhagens mostraramse suscetíveis aos isolados provenientes da Zona da Mata e Agreste, que foram considerados mais virulentos. As cultivares e linhagens que se mostraram resistentes ao isolado proveniente do Sertão foram: IPA-10, Corrente, Mão Curta, Gordo e L-96029, sendo este isolado considerado menos virulento.

Palavras-chave adicionais: Phaseolus vulgaris, resistência, podridão do colo.

\section{ABSTRACT \\ Identification of resistance sources in bean to Sclerotium rolfsii}

Collar rot of common bean (Phaseolus vulgaris), caused by Sclerotium rolfsii, can induce high production losses. The control measures, commonly recommended for this disease, have not been providing effective results. The objective of the present work is to identify the sources of resistance to $S$. rolfsii in common beans. The isolates were obtained from the stem of infected plants, coming from different areas of the State of Pernambuco, and the inoculation was done by deposition of ten sclerotia on the collar of previously wounded plants. The severity of the disease was evaluated through note scale varying from 1 to 9 , proposed by the CIAT and the data were transformed into the Mackinney disease index. All the cultivars and lines have shown susceptibility to the isolates from Zona da Mata and Agreste, which were considered more virulent. The cultivars and lines which showed resistance to the isolate from Sertão were: IPA-10, Corrente, Mão Curta, Gordo and L96029, which wer considered less virulent.
A podridão do colo é importante e freqüentemente observada em áreas quentes, sub-tropical e tropical onde se cultiva o feijoeiro (Phaseolus vulgaris L.). Foi relatada em vários países como Brasil, Bolívia, Equador, Colômbia, Venezuela, Costa Rica, México e EUA (Abawi \& PastorCorrales, 1990); Austrália, Japão, Ceilão, Cuba, Havaí e Filipinas (Weber, 1931). Essa doença é causada por Sclerotium rolfsii Sacc. que sobrevive no solo sob forma de escleródios por períodos de um a três anos (Punja, 1985). Possui capacidade de competição saprofítica e produz elevado número de escleródios (Cardoso, 1994), tornando difícil seu controle.

Medidas de controle são estudadas para reduzir os prejuízos, tais como o controle biológico, principalmente com Trichoderma spp. (Benhamou \& Chet, 1996); com extratos vegetais (Valarini et al., 1991); com solarização do solo (Ghini et al., 1993); com tratamento químico de sementes e solo; calagem; aplicação de resíduos orgânicos; aração profunda
(Punja, 1985), e também o uso de cultivares resistentes ou tolerantes (Abawi \& Pastor-Corrales, 1990).

Cultivares resistentes à $S$. rolfsii são importantes, entretanto não é usual encontrar genes condicionando resistência para patógenos que têm ampla gama de hospedeiros. Resistência para $S$. rolfsii e muitos fungos fitopatógenos de plântulas é rara (Nelson, 1977), e poucas informações de reação de germoplasma de feijão para infecção de $S$. rolfsii estão disponíveis (Abawi \& Pastor-Corrales,1990).

Este trabalho objetivou identificar fontes de resistência à $S$. rolfsii, onde se avaliaram 20 cultivares utilizadas por agricultores da região e dez linhagens que estão em fase de avaliação de suas características agronômicas.

Os três isolados utilizados foram provenientes de plantas infetadas das sub-regiões de Pernambuco: Zona da Mata $\left(\mathrm{S}_{1}\right)$, Agreste $\left(\mathrm{S}_{2}\right)$ e Sertão $\left(\mathrm{S}_{3}\right)$, sendo retiradas estruturas do patógeno diretamente do caule de plantas infetadas, transferidas para placas de Petri com Aveia-Ágar (AA) e 
incubadas por dez dias à temperatura ambiente. As 20 cultivares (IPA-6, IPA-8, IPA-9, IPA-10, IPA-11, Princesa, Gordo, Feijão de Cacho, Corrente, Mão Curta, Gurgutuba, Boi Deitado, Pérola, Carioca de Cacho, Rio Tibagi, A-768, A-774, R-27 e H-92) e dez linhagens (AN-9022421, AN9022180, AN-9021469, AN-9021470, AN-9011455, L96029, LM-93204303, LM-93204319, LM-93204496, LM9220225) utilizadas foram semeadas em vasos plásticos, contendo solo esterilizado, ficando quatro plantas por vaso, as quais foram inoculadas quando atingiram 15 dias de idade, a partir da semeadura. A inoculação foi realizada depositandose no colo de cada planta, levemente ferido com estilete flambado, dez escleródios sobre o ferimento. As plantas foram mantidas em câmara úmida por $48 \mathrm{~h}$. As avaliações foram realizadas aos dez e 35 dias após a inoculação, utilizando uma escala de notas descritiva de Schoonhoven \& PastorCorrales (1987). A partir das notas obtidas, foi calculado o índice de doença de Mackinney (1923), segundo a fórmula $\mathrm{ID}=(\mathrm{f} . \mathrm{v}) / \mathrm{n} . \mathrm{x}$ onde, $\mathrm{f}=\mathrm{n}^{\mathrm{o}}$ de plantas em cada categoria, $\mathrm{v}=$ nota de escala, $n=n^{\circ}$ total de plantas e $\mathrm{x}=$ grau máximo de infecção. $\mathrm{O}$ delineamento experimental utilizado foi inteiramente casualizado em arranjo fatorial (três isolados x 20 cultivares e dez linhagens) com quatro repetições por tratamento. A temperatura e umidade relativa do ar durante a condução do experimento variaram de 28,5 a $31{ }^{\circ} \mathrm{C}$ e 79,4 a $82,6 \%$, respectivamente.

Conforme resultados (Tabela 1), verificou-se que todos os isolados foram patogênicos às cultivares e linhagens testadas. As cultivares R-27, A-768 e Rio Tibagi e linhagens LM-93204303, AN-9022421 e LM-9220225 apresentaram reações diferentes ao isolado $\mathrm{S}_{3}$, mostrando que o melhor período de avaliação foi aos 35 dias após inoculação, ou seja, por ocasião do início do enchimento dos grãos, para a maioria das cultivares e linhagens avaliadas. Estes resultados estão de acordo com os apresentados por Schoonhoven \& PastorCorrales (1987), que relatam este estádio do feijoeiro como um dos mais suscetíveis. Os isolados interagiram diferencialmente com as cultivares. Os isolados $\mathrm{S}_{1}$ e $\mathrm{S}_{2}$ mostraramse mais virulentos, enquanto que o isolado $S_{3}$ menos virulento (Tabela 2), induzindo reações de resistência em IPA-10, Corrente, Mão Curta, Gordo e L-96029, destacando-se com menor índice de doença a IPA-10 e L-96029 e reações intermediária e de suscetibilidade nas demais cultivares e linhagens. A presença de interação diferencial indicou que ocorreu especialização do patógeno a nível do hospedeiro e diz-se que a resistência é do tipo vertical (Vanderplank, 1963). Estes resultados mostram a possibilidade do uso dessas cultivares e linhagens como fonte de resistência em cruzamentos para transferência deste caráter para outros genótipos de feijoeiro, a serem utilizadas em regiões de ocorrência do isolado $\mathrm{S}_{3}$.

Observando-se a análise entre cultivar, linhagem e isolados, verificou-se que os isolados $\mathrm{S}_{1}$ e $\mathrm{S}_{2}$ induziram reações de suscetibilidade em todos os materiais testados. Essa prevalência do caráter suscetível já foi constatada por Santos et al. (1998), quando estudaram a reação de 73 genótipos de
TABELA 1 - Reações apresentadas por 20 cultivares e dez linhagens de feijoeiro (Phaseolus vulgaris) inoculadas com três isolados de Sclerotium rolfsii, aos dez e 35 dias após a inoculação

\begin{tabular}{|c|c|c|c|c|c|c|}
\hline \multirow{2}{*}{$\begin{array}{l}\text { Cultivar/ } \\
\text { Linhagem }\end{array}$} & \multicolumn{6}{|c|}{ Isolados de Sclerotium rolfsii } \\
\hline & $\begin{array}{c}\mathrm{S}_{1} \\
(\mathbf{1 A})\end{array}$ & $\begin{array}{c}\mathrm{S}_{1} \\
(2 \mathrm{~A})\end{array}$ & $\begin{array}{c}S_{2} \\
(1 \mathrm{~A})\end{array}$ & $\begin{array}{c}\mathrm{S}_{2} \\
(2 \mathrm{~A})\end{array}$ & $\begin{array}{c}\mathrm{S}_{3} \\
(\mathbf{1 A})\end{array}$ & $\begin{array}{c}\mathrm{S}_{3} \\
(2 \mathrm{~A})\end{array}$ \\
\hline IPA-6 & $\mathrm{S}^{1}$ & $\mathrm{~S}$ & $\mathrm{~S}$ & $\mathrm{~S}$ & $\mathrm{~S}$ & $\mathrm{~S}$ \\
\hline $\mathrm{R}-27$ & S & S & S & S & I & S \\
\hline $\mathrm{H}-92$ & S & S & S & S & S & S \\
\hline IPA-11 & S & S & S & $\mathrm{S}$ & I & I \\
\hline Corrente & S & S & S & S & $\mathrm{R}$ & $\mathrm{R}$ \\
\hline Boi Deitado & $\mathrm{S}$ & S & S & $\mathrm{S}$ & S & $\mathrm{S}$ \\
\hline Carioca de Cacho & S & S & S & $\mathrm{S}$ & S & $\mathrm{S}$ \\
\hline L-96029 & S & S & S & $\mathrm{S}$ & $\mathrm{R}$ & $\mathrm{R}$ \\
\hline AN-9021469 & S & S & S & S & S & $\mathrm{S}$ \\
\hline LM-93204303 & S & S & S & S & I & $\mathrm{S}$ \\
\hline AN-9021455 & S & S & S & S & S & $\mathrm{S}$ \\
\hline Mão Curta & S & S & S & $\mathrm{S}$ & $\mathrm{R}$ & $\mathrm{R}$ \\
\hline A-774 & S & S & S & S & I & I \\
\hline Gordo & S & S & S & S & $\mathrm{R}$ & $\mathrm{R}$ \\
\hline AN-9022421 & S & S & S & S & I & $\mathrm{S}$ \\
\hline Feijão Carioca & S & S & S & S & S & $\mathrm{S}$ \\
\hline AN-9021470 & S & S & S & $\mathrm{S}$ & I & $\mathrm{I}$ \\
\hline Pérola & S & S & S & S & S & $\mathrm{S}$ \\
\hline Rio Tibagi & S & S & S & S & $\mathrm{R}$ & I \\
\hline LM-93204319 & S & S & S & $\mathrm{S}$ & $\mathrm{S}$ & $\mathrm{S}$ \\
\hline IPA-8 & $\mathrm{S}$ & $\mathrm{S}$ & S & $\mathrm{S}$ & I & I \\
\hline Princesa & S & S & S & $\mathrm{S}$ & S & $\mathrm{S}$ \\
\hline A-768 & $\mathrm{S}$ & $\mathrm{S}$ & S & $\mathrm{S}$ & $\mathrm{R}$ & I \\
\hline IPA-10 & S & S & S & S & $\mathrm{R}$ & $\mathrm{R}$ \\
\hline IPA-7 & S & S & $\mathrm{S}$ & $\mathrm{S}$ & I & I \\
\hline AN-9022180 & S & S & S & $\mathrm{S}$ & I & $\mathrm{I}$ \\
\hline LM-9220225 & I & S & S & $\mathrm{S}$ & $\mathrm{R}$ & $\mathrm{S}$ \\
\hline Gurgutuba & I & S & S & $\mathrm{S}$ & I & I \\
\hline LM-93204496 & I & S & S & $\mathrm{S}$ & I & $\mathrm{I}$ \\
\hline IPA-9 & I & $\mathrm{S}$ & $\mathrm{S}$ & $\mathrm{S}$ & I & $\mathrm{I}$ \\
\hline
\end{tabular}

$1 \mathrm{~A}-$ Avaliação aos dez dias após a inoculação

$2 \mathrm{~A}$ - Avaliação aos 35 dias após a inoculação.

${ }^{1} \mathrm{~S}=$ suscetível (ID = 67,88 - 100\%); I = intermediário (ID 34,44 - 66,67\%); $\mathrm{R}=$ resistente $(\mathrm{ID}=0-33,33 \%) . \mathrm{ID}=$ Índice de doença

feijoeiro a S. rolfsii e por Cardona (1967) em estudos de identificação de fontes de resistência em feijoeiro a $S$. rolfsii. O isolado $\mathrm{S}_{3}$ induziu reação de suscetibilidade nas cultivares e linhagens AN-90214555, AN-9021469, Boi Deitado, LM93204303, Princesa, Feijão Carioca, Carioca de Cacho, LM93204319, LM-9220225, AN-9022421, R-27, Pérola, H-92 e IPA-6; e reação intermediária em IPA-9, IPA-7, AN-9022180, IPA-8, AN-9021470, LM-93204496, IPA-11, A-774, Gurgutuba, Rio Tibagi e A-768. A cultivar Rio Tibagi comportou-se como intermediária, divergindo dos resultados apresentados em testes preliminares no CIAT como resistente (Vieira, 1983). Abawi \& Pastor-Corrales (1990) que listam esta cultivar como 
S.A.F. Dantas et al.

resistente, ressaltando que esta reação pode ser para raça e localização específicas. Dessa forma, essa variação de reação pode ser devida a condições do ambiente, metodologias distintas e, provavelmente, diferentes raças do patógeno.

TABELA 2 - Reações apresentadas por 20 cultivares e dez linhagens de feijoeiro (Phaseolus vulgaris) inoculadas com três isolados de Sclerotium rolfsii, aos 35 dias após a inoculação

\begin{tabular}{|c|c|c|c|c|c|c|}
\hline \multirow{2}{*}{ Cultivar/Linhagem } & \multicolumn{6}{|c|}{ Isolados de Sclerotium rolfsii } \\
\hline & $S_{1}$ & $\mathbf{C R}^{1}$ & $\mathbf{S}_{2}$ & $\mathbf{C R}$ & $\mathbf{S}_{3}$ & $\mathbf{C R}$ \\
\hline IPA-6 & $100,00^{2} \mathrm{aA}^{3}$ & $\mathrm{~S}$ & $100,00 \mathrm{aA}$ & $\mathrm{S}$ & 69,13 abcdefB & $\mathrm{S}$ \\
\hline IPA-7 & $86,46 \mathrm{abAB}$ & $\mathrm{S}$ & 96,19 aA & S & 61,31 abcdefB & I \\
\hline IPA-8 & $95,93 \mathrm{abA}$ & S & $93,30 \mathrm{aA}$ & S & 55,75 abcdefB & I \\
\hline IPA-9 & $68,75 \mathrm{abA}$ & S & $100,00 \mathrm{aB}$ & S & 63,70 abcdefB & I \\
\hline IPA-10 & $100,00 \mathrm{aA}$ & $\mathrm{S}$ & $100,00 \mathrm{aA}$ & S & $1,70 \mathrm{hB}$ & $\mathrm{R}$ \\
\hline IPA-11 & $100,00 \mathrm{aA}$ & $\mathrm{S}$ & $100,00 \mathrm{aA}$ & S & 43,47 cdefghB & I \\
\hline Princesa & $94,22 \mathrm{abA}$ & $\mathrm{S}$ & $100,00 \mathrm{aA}$ & S & 93,41 abcA & $\mathrm{S}$ \\
\hline Rio Tibagi & $100,00 \mathrm{aA}$ & S & $100,00 \mathrm{aA}$ & S & 37,06 defghB & I \\
\hline Feijão de cacho & $98,30 \mathrm{abA}$ & $\mathrm{S}$ & $100,00 \mathrm{aA}$ & $\mathrm{S}$ & $93,30 \mathrm{abcA}$ & $\mathrm{S}$ \\
\hline Carioca de cacho & $100,00 \mathrm{aA}$ & S & $100,00 \mathrm{aA}$ & S & 89,67 abcdA & $\mathrm{S}$ \\
\hline Pérola & $98,75 \mathrm{aA}$ & S & $100,00 \mathrm{aA}$ & S & 69,33 abcdefB & $\mathrm{S}$ \\
\hline Corrente & $100,00 \mathrm{aA}$ & S & $100,00 \mathrm{aA}$ & S & $25,00 \mathrm{fghB}$ & $\mathrm{R}$ \\
\hline Mão Curta & $100,00 \mathrm{aA}$ & S & $100,00 \mathrm{aA}$ & S & 27,17 efghB & $\mathrm{R}$ \\
\hline Gordo & $98,30 \mathrm{abA}$ & S & $100,00 \mathrm{aA}$ & $\mathrm{S}$ & $25,20 \mathrm{fghB}$ & $\mathrm{R}$ \\
\hline Gurgutuba & $79,96 \mathrm{abB}$ & S & $100,00 \mathrm{aA}$ & S & 37,06 defghC & I \\
\hline Boi Deitado & $100,00 \mathrm{aA}$ & S & $100,00 \mathrm{aA}$ & S & $96,19 \mathrm{abA}$ & $\mathrm{S}$ \\
\hline A-768 & $98,97 \mathrm{aA}$ & S & $100,00 \mathrm{aA}$ & $\mathrm{S}$ & 34,80 defghB & I \\
\hline A-774 & $100,00 \mathrm{aA}$ & S & $89,67 \mathrm{aA}$ & S & 37,06 defghB & I \\
\hline $\mathrm{R}-27$ & $100,00 \mathrm{aA}$ & S & $100,00 \mathrm{aA}$ & S & 72,32 abcdefB & $\mathrm{S}$ \\
\hline H-92 & $100,00 \mathrm{aA}$ & $\mathrm{S}$ & $100,00 \mathrm{aA}$ & $\mathrm{S}$ & 69,13 abcdefB & $\mathrm{S}$ \\
\hline AN-9021455 & $100,00 \mathrm{aA}$ & S & $100,00 \mathrm{aA}$ & S & $98,30 \mathrm{aA}$ & $\mathrm{S}$ \\
\hline LM-93204319 & $98,02 \mathrm{abA}$ & S & $100,00 \mathrm{aA}$ & S & 89,67 abcdA & $\mathrm{S}$ \\
\hline AN-9021469 & $100,00 \mathrm{aA}$ & S & $100,00 \mathrm{aA}$ & S & $98,30 \mathrm{aA}$ & $\mathrm{S}$ \\
\hline LM-9220225 & $80,44 \mathrm{abB}$ & S & $99,80 \mathrm{aA}$ & S & 88,14 abcdAB & $\mathrm{S}$ \\
\hline AN-9022421 & $98,30 \mathrm{abA}$ & S & $100,00 \mathrm{aA}$ & $\mathrm{S}$ & 85,35 abcdeB & $\mathrm{S}$ \\
\hline AN-9021470 & $99,80 \mathrm{aA}$ & S & $100,00 \mathrm{aA}$ & $\mathrm{S}$ & 48,26 bcdefgB & I \\
\hline LM-93204496 & $100,00 \mathrm{aA}$ & S & $100,00 \mathrm{aA}$ & S & 43,47 cdefghB & I \\
\hline AN-9022180 & $97,60 \mathrm{abA}$ & $\mathrm{S}$ & $100,00 \mathrm{aA}$ & $\mathrm{S}$ & 56,53 abcdefB & I \\
\hline LM-93204303 & $100,00 \mathrm{aA}$ & S & $100,00 \mathrm{aA}$ & $\mathrm{S}$ & $96,19 \mathrm{abA}$ & $\mathrm{S}$ \\
\hline L-96029 & $100,00 \mathrm{aA}$ & S & $100,00 \mathrm{aA}$ & S & $3,81 \mathrm{ghB}$ & $\mathrm{R}$ \\
\hline
\end{tabular}

\section{REFERÊNCIAS BIBLIOGRÁFICAS}

ABAWI, G.S. \& PASTOR-CORRALES, M.A. Root rots of beans in Latin America and Africa: diagnoses, research methodologies and management strategies. Colômbia. CIAT. 1990.

BENHAMOU, N. \& CHET, I. Parasitism of sclerotia of Sclerotium rolfsii by Trichoderma harzianum: ultrastructural and cytochemical aspects of the interaction. Phytopathology 86:405416. 1996.

CARDONA, C. Doenças. In: Vieira, C. (Ed.) O feijoeiro comum, cultura, doenças e melhoramento. Viçosa-MG. 1967. pp.84124
CARDOSO, J.E. Podridão do colo. In: Sartorato, A. \& Rava, C.A. (Eds.). Principais doenças do feijoeiro comum e seu controle. Brasília. EMBRAPA-SPI. 1994. pp.165-172.

GHINI, R., BETTIOL, W. \& CALDARI JUNIOR, P. Solarização do solo para o controle de Sclerotium rolfsii em feijoeiro. Summa Phytopathology 19:39. 1993. (Resumo).

MACKINNEY, R.H. Influence on soil temperature and moisture on infection of wheat seedlings by Helminthosporium sativus. Journal of Agricultural Research 26:195-218. 1923.

NELSON, R.R. The limits of disease control by genetic means. In: Nelson, R.R. (Ed.). General considerations, concepts, and terminology. $2^{\text {nd }}$ ed. Pennsylvania: The Pennsylvania State 
University Press, 1977. pp. 67-73.

PUNJA, Z.K. The biology, ecology and control of Sclerotium rolfsii. Annual Review of Phytopathology 23:97-127. 1985.

SANTOS, S.C., CHAVES, K.C. \& COSTA, J.L. da. Reação do feijoeiro a Sclerotium rolfsii Sacc., agente causal da podridão do colo. Fitopatologia Brasileira 23:280. 1998. (Resumo).

SCHOONHOVEN, A. van. \& PASTOR-CORRALES, M.A. Standard system for the evaluation of bean germplasm. Colômbia, CIAT. 1987.

VALARINI, P.J., MELO, I.S., FRIGHETTO, R.T.S. \& FERRACINI,
V.L. Avaliação de extratos vegetais no controle de fitopatógenos. Seminário sobre Pragas e Doenças do Feijoeiro. Campinas (SP). Instituto Biológico de São Paulo. 1991. (Resumo)

VANDERPLANK, J.E. Plant disease epidemics and control. New York. Academic Press. 1963.

VIEIRA, C. Doenças e pragas do feijoeiro. Viçosa, UFV. 1983.

WEBER, G.F. Blight of carrots caused by Sclerotium rolfsii, with geographic distribution and host range of the fungus. Phytopathology 21:1129-1140. 1931. 